Yustas, Laura.

Doctoranda en el programa Arte: Producción e Investigación, en Universidad Politécnica de Valencia, Departamento de Escultura.

\title{
Conocimiento situado y epistemología feminista en la inves- tigación en arte. \\ De cómo defender en una comunicación los conflictos específicos del uso de la enunciación académica en la investigación en arte.
}

\author{
TIPO DE TRABAJO
}

Comunicación.

PALABRAS CLAVE

Conocimiento situado, epistemología feminista, investigación en arte, crítica al método científico.

KEY WORDS

Situated knowledge, feminist epistemology, art research, critical to the scientific method.

RESUMEN

Esta comunicación es un acercamiento a un debate complejo y específico de la investigación en arte, que es el de las contradicciones que derivan del uso del método científico como base de la legitimación de un conocimiento (tanto textual y académico como multi e interdisciplinar) fuertemente atravesado por las críticas contemporáneas a la teoría del conocimiento. En este sentido, el debate toma forma de choque epistemológico, ontológico y metodológico, en un terreno en que conviven el método científico basado en el paradigma positivista y la concepción del conocimiento (de las obras de arte como objeto de conocimiento) como construcción simbólica y semántica de carácter social y político. Entendiendo lo textual como un soporte susceptible de ser intervenido y con grandes potencialidades más allá de la mera traducción descriptiva de las experiencias y los hallazgos, me cuestiono los porqués y paraqués de ceñirse al academicismo a la hora de poner en palabras la investigación en arte. En este sentido, pretendo invitar a la reflexión acerca de los modos de enunciar en la producción académica y al planteamiento del texto como espacio de experimentación capaz (o no) de recoger las especificidades y la riqueza de este tipo de investigaciones.

Por tanto, y para abordar lo que podríamos considerar una crisis de los modos de legitimación del conocimiento específicos de la producción cultural, defiendo la crítica feminista a la epistemología a través del concepto de conocimiento situado, apostando por las genealogías, por la irrupción de los cuerpos, las subjetividades y la diversidad en los espacios específicos de producción de estos saberes.

\section{ABSTRACT}

This text is an approach to a complex and specific discussion of research in art, which is the one about the contradictions arising from the use of the scientific method as the basis of legitimacy of a knowledge (textual, academic and multidisciplinary), that is heavily pierced by contemporary theory of knowledge criticism. In this sense, the debate takes the form of an epistemological, ontological and methodological shock, in an area where the scientific method based on the positivist paradigm and the concept of knowledge (of works of art as an object of knowledge) live together as a symbolic and semantic construction with social and political characteristics. If we understand the text as a likely stand to be intervened and we understand that it has great potential beyond mere descriptive translation of the experiences and findings, I wonder the reasons and motivations to stick to academicism when putting into words the art research. Given this situation, I intend to invite to reflect on ways of stating in academic production and the approach of the text as a space for experimentation that can (or not) cluster the specificities and richness of this type of research.

Therefore, and in order to address what might be considered a crisis of legitimacy of specific modes of cultural production of knowledge, I stand up for the feminist critique of epistemology through the concept of situated knowledge, relying on the genealogies, the emergence of bodies, subjectivities and diversity in specific areas of production of this field of knowledgement. 


\section{CONTENIDO}

\section{ESTRUCTURA}

Esta comunicación se estructura en relación a CUATRO cuestiones que se desarrollarán a continuación: 1 . El conocimiento situado como elemento transformador de las formas de entender y legitimar los saberes; 2 . Mi posición en el debate y el origen de mi interés por él; 3. El estado actual del debate en torno a la investigación en arte; y 4. La utilidad social como criterio para la legitimación de la investigación.

\section{CONOCIMIENTO SITUADO, EPISTEMOLOGÍA FEMINISTA Y ENUNCIACIÓN}

Considero necesario comenzar introduciendo el concepto de conocimiento situado, de modo que me referiré a algunas de las autoras que han trabajado en torno él y a las consecuencias que sus postulados tienen sobre consideraciones fundamentales en la investigación científica, como son la pretensión de neutralidad y la de objetividad.

Al hablar de conocimiento situado ${ }^{1}$, se hace referencia a un posicionamiento político desde el cual la subjetividad es considerada como un valor y no como una traba que hace menos válidos los juicios. Considerando que se puede generar conocimiento a partir de la subjetividad y de la praxis que atraviesa los cuerpos, y no sólo desde la teórica objetividad académica, se afirma y reconoce que el conocimiento es un bien común. Supone, en este sentido, una fuerte crítica a la epistemología positivista por su planteamiento de la objetividad entendida en sentido estricto no sólo como una posibilidad indeseable, sino como una imposibilidad práctica. Ha venido a denominarse epistemología feminista. Ello tiene profundas implicaciones en la investigación, ya que parte de la consideración de que la realidad no existe per se, sino que se construye en relación a lo que somos y, a su vez, nos construye. La forma de alcanzar los mejores resultados en la investigación deja de ser, por tanto, generar la máxima neutralidad de los espacios de investigación para "revelar" las leyes internas que rigen el mundo -desde un punto de vista claramente positivista- para, a continuación, buscar la manera más "objetiva" de transmitir esos hallazgos. Muy al contrario, al considerar que la realidad externa no es necesariamente fija y que, en cualquier caso, no puede alcanzarse, se pone de relevancia la capacidad significante de los sujetos, valorizándose el análisis y la investigación de los significados y sentidos que las personas otorgamos a lo que nos rodea y que, a fin de cuentas, conforma lo real. A nivel de enunciación y de transmisión de los saberes generados, el trabajo desde el conocimiento situado, requiere tener en consideración algunos factores que pueden tirar por tierra el trabajo anterior y que igualmente han sido abordados desde los feminismos por autoras como Donna Haraway ${ }^{2}$ o Sandra Harding ${ }^{3}$ y empleados por diversos colectivos ${ }^{4}$. Entre ellos, encontramos los recursos lingüísticos específicos trabajados junto a las metodologías cuantitativas en las ciencias sociales, como son la personificación de datos y estadísticas como si "hablasen por sí mismas", sin mediación de la personas que investiga, o la apelación a lugares comunes a través de expresiones como "es evidente que" o "no cabe duda de que". Algunas autoras feministas de la Tercera Ola, como Aurora Levins Morales, han recuperado esta cuestión y la han planteado directamente en relación al texto académico:

"El lenguaje en el que se expresan las ideas nunca es neutro. El lenguaje que usan las personas revela importante información acerca de con quiénes se identifican, cuáles son sus intenciones, para quién están escribiendo o hablando. El envoltorio es la mercantilización del producto y cumple la función exacta para la que se ha diseñado. El lenguaje innecesariamente especializado se utiliza para humillar a quienes se supone que no deben sentirse autorizados para entenderlo. Vende la ilusión de que sólo quienes pueden manejarlo son capaces de pensar." ${ }^{5}$

Además, la autora, un poco más adelante en el mismo texto, hace referencia a su posición política respecto de la lectura de textos académicos que apoyan su legitimidad en la complejidad terminológica privativa:

\footnotetext{
${ }^{1}$ Para una breve revisión del desarrollo del conocimiento situado y del uso de prácticas como la autoconciencia o la investigación militante: Malo de Molina, Marta: "Prólogo". En Posse, et al.: Nociones comunes. Experiencias y ensayos entre investigación y militancia. Traficantes de Sueños, Madrid, 2004, pp. 13-40. Sobre su uso específico en la práctica artística, véase: Raven, Arlene: "Womanhouse"; y Wilding, Faith. "The Feminist Art Programs at Fresno and CalArts, 1970-75", en Broude, Norma \& Garrard, Mary D. (eds.): The Power of Feminist Art. Abrams, New York, 1994.

${ }^{2}$ La autora dedica un capítulo a los conocimientos situados en su libro: Haraway, Donna: Ciencia, cyborgs y mujeres. La reinvención de la naturaleza. Ediciones Cátedra, Madrid, 1995.

${ }^{3}$ Harding, Sandra: Ciencia y feminismo. Ediciones Morada, Madrid, 1996.

${ }^{4}$ Para una visión profunda de procesos colectivos situados recomiendo encarecidamente los siguientes textos: Precarias a la deriva: $A$ la deriva (por los circuitos de la precariedad femenina). Traficantes de sueños, Madrid, 2003; Crabbé, Olivier «Mouss»; Müller, Thierry y Vercauteren, David: Micropolíticas de los grupos. Para una ecologías de las prácticas colectivas. Traficantes de Sueños, Madrid, 2014. ${ }^{5}$ Levins Morales, Aurora: "Intelectual orgánica certificada" en hooks, bell, et al: Otras inapropiables. Feminismos desde las fronteras. Traficantes de Sueños, Madrid, 2004, p. 68.
} 
“(...) Pero mi elección de leer lo legible tiene que ver con un orden de prioridades diferente [no con la pereza]. El lenguaje está ligado al contenido, y el contenido que yo busco es una teoría y una práctica intelectual que me resulte de utilidad en una investigación activista cuyas prioridades son, sobre todo, democratizadoras." ${ }^{6}$

Considero especialmente interesante la reflexión de Levins Morales por su contemporaneidad, por la lucidez de su posición en los feminismos de los márgenes, por su capacidad para valorar su propio criterio y no dejarse embaucar por las críticas o las consideraciones que ha recibido por parte de personas de la universidad (que ella misma menciona en el texto) y porque hace referencia no sólo a su posición como intelectual a la hora de escribir, sino también a que leer críticamente puede significar, en un momento dado, decidir no leer ciertos textos o a ciertas personas. Esto, en gran medida, va contra la lógica de la comprobación de antecedentes en un tema concreto, pero es muy coherente si nos planteamos que, al fin y al cabo, escojamos lo que escojamos siempre hay pensadoras y pensadores que van a quedarse fuera de nuestros análisis. Por tanto, confiando en la capacidad humana para pensar complejamente y para perseverar en la investigación, nos encontramos con un relativismo que es útil a la hora de analizar por qué unos autores (especialmente en masculinos) son ineludibles y otras y otros son sistemáticamente invisibilizados. Estas consideraciones invitan a plantearse hasta qué punto son fundamentales los pilares del conocimiento occidental hegemónico.

Esto es especialmente importante en su aplicación a la interculturalidad y a los procesos de apoyo mutuo dentro de los movimientos emancipatorios, como es el caso del feminismo. Hasta ahora, hemos sido capaces de comprender que no existe una verdad única que descubrir, pero es necesario que, además de abrir nuestras mentes, seamos capaces de empoderarnos y comenzar a autolegitimar nuestros saberes más allá de la academia, de la educación formal y de la Ciencia.

\section{POSICIONAMIENTO Y TESIS DOCTORAL}

En este primer apartado, me interesa hacer un acercamiento a mi posición personal, tomándome un momento para situarme en relación al tema tratado. Este trabajo de evidenciación de mi postura como investigadora es especialmente importante en esta investigación, debido a la búsqueda de ruptura con la pretensión de neutralidad y la exploración de diferentes modos de entender la subjetividad y la objetividad.

Como artista multidisciplinar y doctoranda precaria, me encuentro con una situación compleja y en cierto modo inesperada: mi tesis doctoral o más bien el sentido mismo de realizar una tesis doctoral desde la práctica artística, de tanto en tanto, se me escapa de las manos. Hace años que estoy muy interesada en la producción de conocimiento de manera horizontal, en los procesos de autolegitimación de saberes a través de la praxis y en la importancia de que la investigación pase por los cuerpos en vez de quedarse atascada en la teoría ${ }^{7}$. He transitado colectivamente la producción artística de diversas maneras y, al buscar cómo poner otras formas de legitimación sobre la mesa, he dado con diversos colectivos que han decido establecer sus propias metodologías no generalizables, defendiendo la posibilidad de que experimentar nuevos modelos de investigación igualmente estancos no tiene por qué ser la única solución al desconocimiento de formas de trabajo colectivo (como bien apuntan los autores de Micropolíticas de grupos, recomendados en una nota al pie anterior). Desde el proceso de investigación, lo que se persigue no es demostrar una serie de hipótesis susceptibles de ser aplicadas a cualquier caso similar, sino ofrecer situaciones, contextos, para ayudar a otras personas a multiplicar sus expectativas y las posibilidades de la creación colectiva a través de la ampliación del imaginario de lo común. Se trataría, entonces, de contribuir a la construcción de una cultura de antecedentes en la práctica colectiva que nos ayudara a legitimar nuestras investigaciones a través de otros caminos. Por supuesto, a la hora de enunciar una posición, más allá de su planteamiento como parcial y situado, es importante medir la fuerza de la voz, su modulación y las consecuencias que puede tener en otras voces cercanas que igualmente luchan por ser escuchadas. Como feminista y como artista la forma de enunciar mi punto de vista tiene gran importancia, ya que estas indagaciones acerca del conocimiento situado, me han obligado a ser especialmente consciente y cuidadosa con las relaciones de poder al ejercer la enunciación y con la presentación como idea de verdad de una ficción de verdad útil.

Por tanto, podemos destacar tres cuestiones fundamentales dentro de la preocupación por la investigación artística y, en especial por el formato tesis, en la universidad. Por un lado, estaría el problema de la concepción de la teoría y la práctica como dos entes separados y contrapuestos, así como la infravaloración de la práctica; por otro, la falta de atención a las formas de enunciación y a su condición de ejercicios de poder, que no debe ser tomada a la ligera; y, por último, el hecho de que considerar la objetividad, la neutralidad y la universalidad como criterios de legitimación del conocimiento, se basa en el privilegio masculino, blanco, occidental, heterosexual y-aún hoy- de clase media, que es al fin y al cabo quien "puede" ostentar la posición de sujeto neutro.

Con todo, la contradicción que me sirve de punto de partida reside en la dificultad de encajar mis planteamientos y mi forma de comprender la investigación en el formato específico de la tesis doctoral -con sus características formales, pero especialmente con sus exigencias de cara a la legitimación externa de lo que en ella se defienda-.

\footnotetext{
${ }^{6}$ Ibídem, p. 69.

${ }^{7}$ Para una revisión de la problemática de quedarse en la teoría puede consultarse: A. M. "Teorías (efectos de las)" en Crabbé, Olivier «Mouss»; Müller, Thierry y Vercauteren, David. Op. Cit. pp. 217-228.
} 


\section{INVESTIGACIÓN ARTÍSTICA EN LA UNIVERSIDAD}

En tercer lugar, abordaré una serie de cuestiones que considero clave en el debate actual en torno a la adaptación de la investigación en arte al marco universitario español y al método científico.

La revisión de actas de jornadas y de textos sobre investigación en arte me ha llevado a localizar cuatro puntos "de intensidad" que se repiten en los autores visitados. El primero de ellos es la dificultad que ha conllevado, y aún conlleva, la adaptación de la investigación en bellas artes al contexto universitario, por la necesidad de conjugar su especificidad con el imperativo de fiabilidad característico de la universidad. ${ }^{8}$ El segundo punto clave, es la consideración de que la investigación en arte tiene una serie de especificidades que hacen que sea un error asumir el método científico como forma de legitimación. Las especificidades a las que hace referencia esta afirmación son generalmente tres: la estrecha relación teoría-praxis, la inclusión de la subjetividad y la sensibilidad ${ }^{9}$, y el trabajo directo con la complejidad ${ }^{10}$. El tercer punto es la consideración de que la legitimación de la investigación artística, entendiendo ésta como los procesos y obras resultantes, ya tiene una estructura propia constituida por el mercado del arte, la crítica y las instituciones culturales $^{11}$. Este punto es especialmente cuestionable si tenemos en cuenta el choque entre la función social del arte y el dejar gran parte de la responsabilidad de legitimación a un organismo como el mercado del arte que no atiende en modo alguno a este criterio. El último punto, que encontramos en el cruce de la práctica artística con la epistemología feminista, es la no asunción del método científico como posicionamiento político de implicación con la democratización de los saberes y el empoderamiento colectivo para su producción y legitimación.

En este último punto, que es el fundamental en mi argumentación, podemos encontrar algunas de las dudas fundamentales que, desde mi punto de vista, atraviesan la producción textual académica como forma de legitimación de la capacidad investigadoras de las y los artistas.

A la luz de las cuestiones planteadas, me planteo qué sentido tiene en estos momentos plantear una tesis al uso como parte de una investigación en arte. En cualquier caso, creo que habría que considerar el factor de la utilidad tanto social como individual para valorar adecuadamente la cuestión. Uno de estos factores, sería si la universidad tiene legitimidad democratizadora a pesar de basarse en el método científico, en la escritura académica y de, además, estar dejando de ser accesible para todas las personas debido a su coste. Otra cuestión es si se puede confiar la tarea de discernir entre un conocimiento y otro (en aras de "validarlo") a una institución que se ha apoyado desde sus orígenes en la expropiación de saberes de minorías para generar productos científicos (incluidos los títulos universitarios como producto) exportables y rentables. ${ }^{12}$ Igualmente, surge la duda sobre qué ocurre cuando se llega a la conclusión de que ciertos conocimientos y formas de hacer funcionan mejor alejadas de la universidad, ¿significaría que tienen que "asumir" quedar relegadas fuera de los circuitos académicos? Me pregunto qué implica quedarse fuera cuando dentro no hay posibilidades laborales.

Por último, vuelvo a la cuestión de la enunciación para preguntarme si el uso de lenguaje especializado no tendrá como finalidad, más que ser específico y eficiente, mantener alejados a los ilegitimables, a los que construyen conocimiento desde otros lugares. Esto podría abrir la puerta a la necesidad (y la posibilidad) de crear formas flexibles de legitimación desde dentro de la universidad y desde fuera, planteando modelos que facilitaran el trabajo de los y las investigadoras sin limitar sus investigaciones. Una de ellas, podría ser el criterio de utilidad que expongo a continuación.

\section{QUÉ ES ÚTIL}

En este punto, quisiera defender la utilidad en tanto a criterio de calidad, emancipatorio y autolegitimador que permite la continuación de nuestro desarrollo como personas. Plantearé mi particular visión de la utilidad y el interés específico de plantearse la aplicabilidad de la praxis sobre la teoría y viceversa, siempre teniendo en cuenta la importancia de situarse de un modo crítico frente al capitalismo cognitivo y la actitud agresiva y no proactiva propia de la tecnociencia más salvaje.

Utilidad, por tanto, en relación a dos condicionantes: a la calidad e interés de las propuestas, y a la rigidez de los procesos vitales. Calidad, en tanto a avance hacia la valoración de la diversidad y la consecución de la igualdad en trato, visibilidad y derechos; así como de utilidad para la mejora de la vida de las personas en diversos sentidos (utilidad de las imágenes para el empoderamiento de un colectivo específico, por ejemplo). Rigidez de los procesos vitales, en relación a cómo ha podido cambiar en los últimos años la moti-

\footnotetext{
${ }^{8}$ De Laiglesia y González de Peredo, Juan Fernando; Rodríguez Caeiro, Martín y Fuentes Cid, Sara. (eds.): Notas para una investigación artística. Actas Jornadas La carrera investigadora en Bellas Artes: Estrategias y Modelos (2007-2015). Universidad de Vigo, Servicio de Publicaciones, Vigo, 2008, p. 21.

${ }^{9}$ Ibídem, p. 52.

${ }^{10}$ Ibídem, p. 23.

${ }^{11}$ Ibídem, pp. 77-78.

${ }^{12}$ Para ahondar en la historia de la universidad como institución y en la expropiación de saberes, véase: Federici, Silvia: Calibán y la bruja. Mujeres, cuerpo y acumulación originaria. Traficantes de Sueños, Madrid, 2010.
} 
vación para hacer una investigación de corte científico. Si el horizonte laboral está más que difuso y la posibilidad de un puesto como docente en la universidad es tan improbable como la de un puesto público para la investigación en artes visuales, ¿qué estamos buscando en formatos de tesis doctoral que no encajan en muchos de nuestros planteamientos básicos? Me interesa pensar la utilidad, una vez más, de la ficción de la tesis como prueba de capacidad investigadora en un campo que, metodológicamente, dista mucho del de la tesis.

Es fundamental, igualmente, cuestionar el papel del texto académico como producción "efectiva" de conocimiento para la sociedad ya que el lenguaje que se exige desde la universidad puede llegar a ser increíblemente críptico para "no iniciados" y contribuye en gran medida al ejercicio del poder de la enunciación sobre colectivos no legitimados por la institución universitaria. ¿Tiene sentido buscar la excelencia, la exclusividad en el uso de la palabra? En este punto, me parece importante matizar que no considero que el debate se apoye sobre la complejidad, sino sobre la tecnicidad del lenguaje académico. La opción de ser claros y transmitir la complejidad pretendiendo que quien recibe se acerque al sentido que manejamos, es eso, una opción, y su corte es político, de género, de raza, religioso y de clase.

\section{CONCLUSIONES}

Con esta comunicación no pretendía dar todas las respuestas, sino más bien plantear una serie de dudas que reconozco como lugares comunes en la investigación en arte. Desearía que este texto, al igual que textos anteriores con los que he podido reflexionar, aporte complejidad y al mismo tiempo claridad al debate ya que, desde mi punto de vista, si algo es necesario en momentos de impasse sociopolítico y económico como el actual, es arrojar claridad sobre las oscuridades que nos impiden trabajar colectivamente en dirección a otros lugares.

Quería, con todo ello, continuar abriendo un camino que tiene parte de ético y parte de práctico. Al fin y al cabo, en teoría sería más sencillo limitarse a cumplir con la ficción de la tesis doctoral (a pesar de que choque con los tiempos y las fuerzas de que en ocasiones disponemos), pero no estamos ante una cuestión de comodidad o sencillez, sino ética, de reconocimiento de la práctica artística y de emancipación. La cotidianeidad apremia y la democratización pierde sentido cuando sólo puede producir quien dispone de capital simbólico, temporal y económico para hacerlo.

\section{FUENTES REFERENCIALES.}

DE LAIGLESIA Y GONZÁLEZ DE PEREDO, Juan Fernando; RODRÍGUEZ CAEIRO, Martín; FUENTES CID, Sara (eds.): Notas para una investigación artística. Actas Jornadas La carrera investigadora en Bellas Artes: Estrategias y Modelos (2007-2015). Universidad de Vigo, Servicio de Publicaciones, Vigo, 2008.

CRABBÉ, Olivier «Mouss»; MÜLLER, Thierry y VERCAUTEREN, David: Micropolíticas de los grupos. Para una ecologías de las prácticas colectivas. Traficantes de Sueños, Madrid, 2014.

FEDERICl, Silvia: Calibán y la bruja. Mujeres, cuerpo y acumulación originaria. Traficantes de Sueños, Madrid, 2010.

HARAWAY, Donna: Ciencia, cyborgs y mujeres. La reinvención de la naturaleza. Ediciones Cátedra, Madrid, 1995.

HARDING, Sandra: Ciencia y feminismo. Ediciones Morada, Madrid, 1996.

HERNÁNDEZ HERNÁNDEZ, Fernando; PÉREZ LÓPEZ, Héctor Julio; GÓMEZ MUNTANÉ, Maricarmen: Bases para un debate sobre investigación artística. Ministerio de Educación y Ciencia, Madrid, 2006.

LEVINS MORALES, Aurora: "Intelectual orgánica certificada" en VVAA. Otras inapropiables. Feminismos desde las fronteras. Traficantes de Sueños, Madrid, 2004, pp. 63-70.

MALO DE MOLINA, Marta: "Prólogo". En Posse, et al.: Nociones comunes. Experiencias y ensayos entre investigación y militancia. Traficantes de Sueños, Madrid, 2004, pp. 13-40.

PRECARIAS A LA DERIVA: A la deriva (por los circuitos de la precariedad femenina). Traficantes de sueños, Madrid, 2003.

RAVEN, Arlene: "Womanhouse"; y WILDING, Faith. "The Feminist Art Programs at Fresno and CalArts, 1970-75", en BROUDE, Norma \& GARRARD, Mary D. (eds.). The Power of Feminist Art. New York: Abrams, 1994. 